MATHEMATICS OF COMPUTATION

Volume 72, Number 244, Pages 1747-1767

S 0025-5718(03)01524-2

Article electronically published on April 29, 2003

\title{
WEAKLY NONOSCILLATORY SCHEMES FOR SCALAR CONSERVATION LAWS
}

\author{
KIRILL KOPOTUN, MARIAN NEAMTU, AND BOJAN POPOV
}

\begin{abstract}
A new class of Godunov-type numerical methods (called here weakly nonoscillatory or WNO) for solving nonlinear scalar conservation laws in one space dimension is introduced. This new class generalizes the classical nonoscillatory schemes. In particular, it contains modified versions of MinMod and UNO. Under certain conditions, convergence and error estimates for WNO methods are proved.
\end{abstract}

\section{INTRODUCTION}

We are interested in the scalar hyperbolic conservation law

$$
\begin{cases}u_{t}+f(u)_{x}=0, & (x, t) \in \mathbb{R} \times(0, \infty), \\ u(x, 0)=u_{0}(x), & x \in \mathbb{R},\end{cases}
$$

where $f$ is a given flux function. In recent years, there has been enormous activity in the development of the mathematical theory and in the construction of numerical methods for (1). Even though the existence-uniqueness theory of weak solutions is complete, there are many numerically efficient methods for which the questions of convergence and error estimates are still open. For example, the original MinMod, UNO, ENO, and WENO methods are known to be numerically robust, at least for piecewise smooth initial data $u_{0}$, but theoretical results about convergence are still missing [3, 7, 8, 20].

In this paper, we consider a class of the so-called Godunov-type schemes for solving (11), see 22]. There are two main steps in such schemes: evolution and projection. In the original Godunov scheme, the projection is onto piecewise constant functions - the cell averages. In the general Godunov-type method, the projection is onto piecewise polynomials. To determine the properties of a scheme it is necessary to study the properties of the projection operator. For example, it is important to know whether this operator reproduces polynomials of a given degree and whether it is nonoscillatory. A numerical method is called nonoscillatory if the number of extrema of the approximate solution does not increase in time. (This method is sometimes referred to as Number of Extrema Diminishing or NED.)

Received by the editor March 1, 2001 and, in revised form, January 28, 2002.

2000 Mathematics Subject Classification. Primary 65M15; Secondary 35L65, 35B05, 35B30.

Key words and phrases. Scalar conservation laws, Godunov-type schemes, error estimates, weak nonoscillation, relaxed entropic projection.

The first author was supported by NSERC of Canada and by NSF of USA under grant DMS9705638 .

The second author was supported by NSF under grant DMS-9803501.

The third author was supported by the ONR Grant No. N00014-91-J-1076. 
Many well-known methods (e.g., MinMod, UNO, and some MUSCL schemes) are nonoscillatory, see [4, 5, 7, 9, 10, 14, 15. The NED property is an invariant of the exact solution operator (this was first formulated in [15]), and all nonoscillatory methods are build to preserve that property. However, nonoscillation is, in general, not sufficient for convergence of such methods to the entropy solution, and more restrictions on the projection step are needed. For example, one can impose the socalled entropy inequalities [1, 2, 18, or require that the projection step be entropy diminishing [5]. Alternatively, for a convex flux, one can impose Lip + stability on the projection and then prove convergence via Tadmor's Lip' theory [17, 21].

In this paper, the notion of weakly nonoscillatory (WNO) schemes is introduced, which is a generalization of the classical concept of nonoscillation. For example, any Godunov-type scheme with nonoscillatory evolution and projection is WNO. We will restrict our attention to Godunov-type methods with exact evolution. A convergence result in this case is important, since it is a key ingredient in the proof of convergence of the fully discrete schemes. Our main result is a convergence theorem for a subclass of WNO Godunov-type schemes (which, in particular, contains modified versions of MinMod and UNO). We derive error estimates by relaxing the classical entropy inequalities imposed on the projection operator [1]. In particular, we prove convergence for such relaxed entropic WNO schemes, provided $f \in \operatorname{Lip}\left(1, L^{\infty}\right)$ and $u_{0}$ is a compactly supported function of bounded variation belonging to the class $\mathcal{W}_{L}, L \in \mathbb{N}$, of weakly nonoscillating functions. This new approach allows us to obtain an error estimate (which depends on $L$ ) for a class of schemes which, in general, do not satisfy the entropy inequalities in [1, 2, 18, [5]. More general results for noncompactly supported initial conditions and fully discrete schemes will be given elsewhere.

The paper is organized as follows. In Section 2, we introduce the class $\mathcal{W}_{L}$, define WNO Godunov-type schemes, and state our main result (Theorem 2). In Section 3, we establish various properties of functions in $\mathcal{W}_{L}$, and then, in Section 4 , we show that the entropy solution of the conservation law preserves this class. Section 5 contains the proof of Theorem 2. Finally, in Section 6, we give examples of WNO schemes whose convergence is guaranteed by Theorem 2, including simple modifications of MinMod and UNO. We also give an example of a scheme that is not relaxed entropic, satisfies all other requirements, and converges to a weak solution, which is not the entropy solution. This shows that the condition that the method is relaxed entropic is essential.

\section{Preliminaries}

Consider the initial value problem

$$
\begin{cases}u_{t}+f(u)_{x}=0, & (x, t) \in \mathbb{R} \times(0, T), \\ u(x, 0)=u_{0}(x), & u_{0} \in L_{l o c}^{1}(\mathbb{R}),\end{cases}
$$

where $T>0$ and

$$
f \in \operatorname{Lip}\left(1, L^{\infty}\right):=\left\{f \in L^{\infty}(\mathbb{R}) \mid \sup _{t>0}\left\{t^{-1} \sup _{0<h \leq t}\|f(\cdot+h)-f(\cdot)\|_{L^{\infty}(\mathbb{R})}\right\}<\infty\right\} .
$$

A function

$$
\begin{aligned}
& u \in C\left((0, T], L_{l o c}^{1}(\mathbb{R})\right):=\left\{u: \mathbb{R}^{2} \rightarrow \mathbb{R} \mid u(t, \cdot) \in L_{l o c}^{1}(\mathbb{R}), t \in(0, T],\right. \\
&\left.\lim _{t^{\prime} \rightarrow t}\left\|u(t, \cdot)-u\left(t^{\prime}, \cdot\right)\right\|_{L_{l o c}^{1}(\mathbb{R})}=0\right\}
\end{aligned}
$$


is called the entropy solution of (2) if

$$
\begin{aligned}
-\int_{0}^{T} & \int_{\mathbb{R}}\left(|u-c| \varphi_{t}+\operatorname{sgn}(u-c)(f(u)-f(c)) \varphi_{x}\right) d x d t \\
& \quad+\int_{\mathbb{R}}|u(x, T)-c| \varphi(x, T) d x-\int_{\mathbb{R}}\left|u_{0}(x)-c\right| \varphi(x, 0) d x \leq 0,
\end{aligned}
$$

for all $c \in \mathbb{R}$ and all nonnegative continuously differentiable functions $\varphi=\varphi(x, t)$, compactly supported on $\mathbb{R} \times \mathbb{R}_{+}$. While there can be many weak solutions, it is well known that the entropy solution of (2) is unique (see [11]).

A function $g$ is of bounded variation, i.e., $g \in \mathrm{BV}(\mathbb{R})$, if

$$
|g|_{\mathrm{BV}(\mathbb{R})}:=\sup \sum_{i=1}^{n-1}\left|g\left(x_{i+1}\right)-g\left(x_{i}\right)\right|<\infty,
$$

where the supremum is taken over all finite sequences $x_{1}<\cdots<x_{n}$ in $\mathbb{R}$. Functions of bounded variation have at most countably many discontinuities, and the left and right limits $g\left(x^{-}\right)$and $g\left(x^{+}\right)$exist at each point $x \in \mathbb{R}$.

Since the values of the initial condition $u_{0}$ on a set of measure zero have no influence on the entropy solution of (2), it is more desirable to replace the seminorm $|\cdot|_{\mathrm{BV}(\mathbb{R})}$ by a similar quantity independent of the function values on sets of measure zero. The standard approach is to consider the $\operatorname{space} \operatorname{Lip}\left(1, L^{1}\right)$ of all functions $g \in L^{1}(\mathbb{R})$ such that the seminorm

$$
|g|_{\operatorname{Lip}\left(1, L^{1}\right)}:=\limsup _{t>0} \frac{1}{t} \int_{\mathbb{R}}|g(x+t)-g(x)| d x
$$

is finite. It is clear that $|g|_{\operatorname{Lip}\left(1, L^{1}\right)}$ will not change if $g$ is modified on a set of measure zero. At the same time, the above two seminorms are equal in the following sense. Every $g \in \operatorname{Lip}\left(1, L^{1}\right)$ can be corrected on a set of measure zero to a function $\bar{g} \in \mathrm{BV}(\mathbb{R})$. Note that any two such corrections can only differ at countably many points. In particular, if this correction $\bar{g}$ is such that $\bar{g}(x)$ lies between $\bar{g}\left(x^{+}\right)$ and $\bar{g}\left(x^{-}\right)$, for all $x \in \mathbb{R}$ where $\bar{g}$ is discontinuous, then $|\bar{g}|_{\mathrm{BV}(\mathbb{R})}=|\bar{g}|_{\operatorname{Lip}\left(1, L^{1}\right)}=$ $|g|_{\operatorname{Lip}\left(1, L^{1}\right)}$ (see [6. Theorem 9.3]). For our purposes, it will be convenient to consider the specific correction satisfying $\bar{g}(x)=\max \left\{\bar{g}\left(x^{+}\right), \bar{g}\left(x^{-}\right)\right\}, x \in \mathbb{R}$. It is easy to show that this $\bar{g}$ is the unique upper semicontinuous (u.s.c.) correction of $g$ such that $|\bar{g}|_{\mathrm{BV}(\mathbb{R})}=|g|_{\operatorname{Lip}\left(1, L^{1}\right)}$. From now on, we refer to this function $\bar{g}$ as the u.s.c. correction of $g$. For later use, we remark that if $g$ is a piecewise polynomial function, then $\bar{g}$ may differ from $g$ only at the points of discontinuity of $g$, and $|\bar{g}|_{\mathrm{BV}(\mathbb{R})}=|g|_{\operatorname{Lip}\left(1, L^{1}\right)}$.

If $f \in \operatorname{Lip}\left(1, L^{\infty}\right)$, then it is well known that the entropy solution of (2) is total variation diminishing (TVD), i.e.,

$$
|u(\cdot, t)|_{\operatorname{Lip}\left(1, L^{1}\right)} \leq\left|u_{0}\right|_{\operatorname{Lip}\left(1, L^{1}\right)}, \quad t>0 .
$$

In order to describe a version of Kuznetsov's error estimate for Godunov-type methods, needed later, we introduce the following notation. Let $\eta \in C^{\infty}(\mathbb{R})$ be such that $\eta \geq 0, \operatorname{supp}(\eta) \subset[-1,1], \int_{\mathbb{R}} \eta(x) d x=1$, and $\eta(x)=\eta(-x)$ for all $x \in \mathbb{R}$. We define

$$
\eta_{\varepsilon}:=\frac{1}{\varepsilon} \eta\left(\frac{\cdot}{\varepsilon}\right), \quad \varepsilon>0,
$$


and

$$
\rho_{\varepsilon}(g, h):=\int_{\mathbb{R}^{2}} \eta_{\varepsilon}(x-y)|g(x)-h(y)| d x d y, \quad g, h \in L^{1}(\mathbb{R}) .
$$

Suppose that $u$ is the entropy solution of (21) corresponding to the initial data $u_{0} \in \operatorname{Lip}\left(1, L^{1}\right)$. Let $N \geq 1$ and $0=t_{0}<\cdots<t_{N}:=T$. Let $v(x, t)$ be a right-continuous function in $t$ such that, for each $n=0, \ldots, N-1, v$ is an entropy solution of

$$
\begin{cases}u_{t}^{n}+f\left(u^{n}\right)_{x}=0, & (x, t) \in \mathbb{R} \times\left(t_{n}, t_{n+1}\right) \\ u^{n}\left(\cdot, t_{n}\right)=v\left(\cdot, t_{n}\right), & v\left(\cdot, t_{n}\right) \in L^{1}(\mathbb{R})\end{cases}
$$

Note that $v$ is uniquely determined by the functions $\left\{v\left(\cdot, t_{n}\right)\right\}_{n=0}^{N-1}$. With this notation, let us recall the following result.

Theorem 1 (Kuznetsov [12]). Let $u$ be the entropy solution of (2) with initial condition $u_{0} \in \operatorname{Lip}\left(1, L^{1}\right)$, and let $v$ be as above. Then,

$$
\begin{gathered}
\left\|v\left(\cdot, t_{N}\right)-u\left(\cdot, t_{N}\right)\right\|_{L^{1}(\mathbb{R})} \leq\left\|u_{0}-v_{0}\right\|_{L^{1}(\mathbb{R})}+2 \varepsilon\left|u_{0}\right|_{\operatorname{Lip}\left(1, L^{1}\right)} \\
\quad+\sum_{n=1}^{N}\left[\rho_{\varepsilon}\left(v\left(\cdot, t_{n}\right), u\left(\cdot, t_{n}\right)\right)-\rho_{\varepsilon}\left(v\left(\cdot, t_{n}^{-}\right), u\left(\cdot, t_{n}\right)\right)\right],
\end{gathered}
$$

for any $\varepsilon>0$.

Note that, by density arguments, this theorem still holds for $\eta=\frac{1}{2} \chi_{[-1,1]}$, where $\chi_{A}$ denotes the characteristic function of a set $A$. In the original Godunov method, $v\left(\cdot, t_{n}\right)$ is the average of $v\left(\cdot, t_{n}^{-}\right)$on $I_{j}:=[j h,(j+1) h), h>0, j \in \mathbb{Z}$, where $v\left(\cdot, t_{0}^{-}\right):=u_{0}$. For a general Godunov-type method, $v\left(\cdot, t_{n}\right)$ is determined from $v\left(\cdot, t_{n}^{-}\right)$by $v\left(\cdot, t_{n}\right):=P_{h} v\left(\cdot, t_{n}^{-}\right)$, where $P_{h}: L^{1}(\mathbb{R}) \rightarrow L^{1}(\mathbb{R})$ is a "projection" operator. For a function $g \in L^{1}(\mathbb{R}), P_{h} g$ is usually a "simpler" function that makes it possible to solve (2) exactly with initial data $P_{h} g$ for small time. For the sake of simplicity, only regular grids are considered in this paper, the results for the nonregular case being analogous.

The properties of a Godunov-type scheme are largely determined by the projection $P_{h}$. Typically, $P_{h}$ should have the following properties (see [22]):

(i) Conservation: For every $g \in L^{1}(\mathbb{R}), \int_{I_{j}} P_{h} g d x=\int_{I_{j}} g d x, j \in \mathbb{Z}$.

(ii) Accuracy: If $g$ is "smooth", then $P_{h} g$ provides good local approximation. For example, if $P_{h} g$ is a polynomial function on $I_{j}$, then it is natural to require that $\left\|P_{h} g-g\right\|_{L^{\infty}\left(I_{j}\right)}$ is of optimal order.

(iii) Boundedness of Total Variation: $P_{h}$ should be such that the total variation of the numerical solution is bounded. That is, $\left|v\left(\cdot, t_{n}\right)\right|_{\operatorname{Lip}\left(1, L^{1}\right)}, n=$ $0, \ldots, N$, are uniformly bounded (independently of $N$ ). This guarantees convergence to a weak solution of (2), see e.g., [13].

In this context, we mention the following so-called high resolution methods (popularized by their three-letter acronyms): the piecewise parabolic method (PPM) [4, the uniformly nonoscillatory (UNO) scheme [7], and the essentially nonoscillatory schemes (ENO) 8]. The implicit assumption in such methods is that an approximation to a piecewise smooth solution is sought with finitely many oscillations and local dependence on the initial data. 
The Godunov-type schemes considered in this paper are such that the projection operator $P_{h}$ meets the following requirements:

(P1) $P_{h}$ is conservative:

$$
\int_{I_{j}} P_{h} g d x=\int_{I_{j}} g d x, \quad g \in L^{1}(\mathbb{R}), \quad j \in \mathbb{Z} .
$$

(P2) $P_{h}$ is TVB (total variation bounded):

$$
\left|P_{h} g\right|_{\operatorname{Lip}\left(1, L^{1}\right)} \leq C_{0}|g|_{\operatorname{Lip}\left(1, L^{1}\right)} .
$$

(P3) $P_{h}$ is local: There exists an $M \in \mathbb{N}$ such that $P_{h} g \equiv 0$ on $I_{j}$ if $g \equiv 0$ on $I_{j}^{M}:=\bigcup_{|j-k| \leq M} I_{k}, j \in \mathbb{Z}$.

(P4) $P_{h}$ is relaxed entropic: There exist constants $\alpha>0$ and $C_{1} \geq 0$ such that

$$
\int_{I_{j}}\left(\left|P_{h} g(x)-\lambda\right|-|g(x)-\lambda|\right) d x \leq C_{1} h^{1+\alpha}|g|_{\operatorname{Lip}\left(1, L^{1}\right)}, \quad g \in \operatorname{Lip}\left(1, L^{1}\right),
$$

for all $j \in \mathbb{Z}$ and all $\lambda \in \mathbb{R}$.

Remark 1. Properties (P1) and (P2) imply that $P_{h}$ has the approximation property

$$
\left\|P_{h} g-g\right\|_{L^{1}(\mathbb{R})} \leq\left(1+C_{0}\right) h|g|_{\operatorname{Lip}\left(1, L^{1}\right)}, \quad g \in \operatorname{Lip}\left(1, L^{1}\right) .
$$

This estimate can be established using the triangle inequality and

$$
\left\|A_{h} g-g\right\|_{L^{1}(\mathbb{R})} \leq h|g|_{\operatorname{Lip}\left(1, L^{1}\right)},
$$

where $A_{h} g$ is the piecewise constant function such that

$$
\left.A_{h} g\right|_{I_{j}}:=\frac{1}{h} \int_{I_{j}} g d x, \quad j \in \mathbb{Z} .
$$

( $A_{h}$ is referred to as the averaging operator later in this paper.) Indeed,

$$
\begin{aligned}
& \left\|P_{h} g-g\right\|_{L^{1}(\mathbb{R})}=\left\|P_{h} g-A_{h}\left(P_{h} g\right)+A_{h}(g)-g\right\|_{L^{1}(\mathbb{R})} \\
& \quad \leq h\left(\left|P_{h} g\right|_{\operatorname{Lip}\left(1, L^{1}\right)}+|g|_{\operatorname{Lip}\left(1, L^{1}\right)}\right) \leq\left(1+C_{0}\right) h|g|_{\operatorname{Lip}\left(1, L^{1}\right)} .
\end{aligned}
$$

Remark 2. If $C_{1}=0$ in (11), then $P_{h}$ is called entropic 1 (or entropy diminishing [5]). For example, the averaging operator $A_{h}$ is entropic.

Remark 3. Inequality (11) is automatically satisfied with $\alpha=0$. This immediately follows from (12) and the triangle inequality. However, this, in general, is not enough for the convergence of the scheme to the entropy solution, see example (E4) in Section 6.

Remark 4. The relaxed entropic condition $(\mathrm{P} 4)$ requires limiters which do not respect the scale invariance $(x, t) \rightarrow(\lambda x, \lambda t)$.

Beside conditions (P1)-(P4), we need some additional restrictions on the numerical scheme. Recall first that a real-valued function $g$ is called upper semicontinuous (u.s.c.) if

$$
\limsup _{y \rightarrow x} g(y) \leq g(x), \quad x \in \mathbb{R} .
$$

As is pointed out above, every $g \in \operatorname{Lip}\left(1, L^{1}\right)$ can be uniquely modified on a set of measure zero to a u.s.c. function $\bar{g} \in \mathrm{BV}(\mathbb{R})$. For $g \in \operatorname{Lip}\left(1, L^{1}\right)$, the level set of $g$ corresponding to $\lambda \in \mathbb{R}$ is defined as

$$
E_{\lambda}(g):=\{x \mid \bar{g}(x)<\lambda\} .
$$


It is well known that the sets $E_{\lambda}(g)$ are open for all $\lambda \in \mathbb{R}$ if and only if $\bar{g}$ is u.s.c. (see [19]). Therefore, each $E_{\lambda}(g)$ can be uniquely represented as a countable union of disjoint open intervals, called the components of $E_{\lambda}(g)$. If the number of such components is finite, then this number is denoted by $\mathcal{L}\left(E_{\lambda}(g)\right)$.

Definition 1. A function $g$ is said to be weakly nonoscillating if it belongs to the class

$$
\mathcal{W}_{L}:=\left\{g \in \operatorname{Lip}\left(1, L^{1}\right) \mid \mathcal{L}\left(E_{\lambda}(g)\right) \leq L, \forall \lambda \in \mathbb{R}\right\}
$$

for some $L \in \mathbb{N}$.

Definition 2. A Godunov-type scheme is called weakly nonoscillatory (WNO) if there exists an integer $L \geq 1$ (independent of $N$ ) such that the approximate solutions $v\left(\cdot, t_{n}\right)$ of (2) are in $\mathcal{W}_{L}$ for all $n=0, \ldots, N$. (To emphasize that a given WNO scheme preserves the class $\mathcal{W}_{L}$ for a specific value of $L$, we refer to this scheme as "WNO with constant $L$ ".)

Definition 3. A Godunov-type scheme is called uniformly bounded if there exists a constant $C_{2}$ such that

$$
\left\|v\left(\cdot, t_{n}\right)\right\|_{L^{\infty}(\mathbb{R})} \leq C_{2}\left\|u_{0}\right\|_{L^{\infty}(\mathbb{R})}
$$

for all $n=0, \ldots, N$.

Remark 5. There are many uniformly bounded schemes (e.g., all TVD schemes). At the same time, there are methods which are numerically uniformly bounded, but rigorous proofs of uniform boundedness are still missing. (We mention UNO as an example.)

Remark 6. One way of obtaining a WNO Godunov-type scheme is to require that the projection $P_{h}$ preserve the class $\mathcal{W}_{L}$. This is because the exact evolution also preserves $\mathcal{W}_{L}$, as shown in Theorem 10 below.

Remark 7. A different possibility to have a WNO Godunov-type scheme is to employ a nonoscillatory projection $P_{h}$. Any such scheme is WNO with $L=[(K+3) / 2]$, where $K$ is the number of local extrema of $u_{0}$, see Theorem 5 . However, it can be WNO with a constant $L^{\prime}$ which is much smaller than $O(K)$, and so preservation of the number of local extrema of the initial condition $u_{0}$ may not be as efficient as preserving the class $\mathcal{W}_{L^{\prime}}$ containing $u_{0}$.

Remark 8. For any $g \in \mathrm{BV}(\mathbb{R})$,

$$
|g|_{\mathrm{BV}(\mathbb{R})} \geq \sup _{\mathbb{R}} g-\inf _{\mathbb{R}} g .
$$

Thus, in particular, if a function $g \in \operatorname{Lip}\left(1, L^{1}\right)$ is compactly supported, then $\operatorname{esssup}_{\mathbb{R}} g \geq 0$ and $\operatorname{essinf}_{\mathbb{R}} g \leq 0$ and, hence,

$$
|g|_{\operatorname{Lip}\left(1, L^{1}\right)} \geq \underset{\mathbb{R}}{\operatorname{esssup}} g-\underset{\mathbb{R}}{\operatorname{essinf}} g \geq\|g\|_{L^{\infty}(\mathbb{R})} .
$$

Remark 9. It is shown in Ziemer [23, Theorem 5.4.4] that, for a function $g \in$ $\operatorname{Lip}\left(1, L^{1}\right)$,

$$
|g|_{\operatorname{Lip}\left(1, L^{1}\right)}=\int_{\mathbb{R}}\left|\chi_{\{x: g(x)>t\}}\right|_{\operatorname{Lip}\left(1, L^{1}\right)} d t .
$$


Hence, if $g \in \mathcal{W}_{L} \cap L^{\infty}(\mathbb{R})$, then

$$
\begin{aligned}
|g|_{\operatorname{Lip}\left(1, L^{1}\right)} & =|-\bar{g}|_{\operatorname{Lip}\left(1, L^{1}\right)}=\int_{\mathbb{R}}\left|\chi_{\{x: \bar{g}(x)<-t\}}\right|_{\operatorname{Lip}\left(1, L^{1}\right)} d t \\
& =\int_{\mathbb{R}}\left|\chi_{E_{\lambda}(g)}\right|_{\operatorname{Lip}\left(1, L^{1}\right)} d \lambda=\int_{\operatorname{essinf}_{\mathbb{R}} g}^{\operatorname{esssup}_{\mathbb{R}} g}\left|\chi_{E_{\lambda}(g)}\right|_{\operatorname{Lip}\left(1, L^{1}\right)} d \lambda \\
& \leq 2 \mathcal{L}\left(E_{\lambda}(g)\right)\left(\underset{\mathbb{R}}{\operatorname{esssup} g-\underset{\mathbb{R}}{\operatorname{essinf}} g) \leq 4 L\|g\|_{L^{\infty}(\mathbb{R})} .}\right.
\end{aligned}
$$

Thus, together with the previous remark, this implies that, if $g \in \mathcal{W}_{L} \cap L^{\infty}(\mathbb{R})$ is compactly supported, then

$$
\|g\|_{L^{\infty}(\mathbb{R})} \leq|g|_{\operatorname{Lip}\left(1, L^{1}\right)} \leq 4 L\|g\|_{L^{\infty}(\mathbb{R})} .
$$

The main result of this paper is the following

Theorem 2. Let $u$ be the entropy solution of (2), where $u_{0}$ is a compactly supported function such that $u_{0} \in \operatorname{Lip}\left(1, L^{1}\right) \cap \mathcal{W}_{L}$, for some $L \in \mathbb{N}$. Also, let $v$ be the numerical solution obtained by a uniformly bounded WNO Godunov-type scheme with constant $L$, satisfying $(\mathrm{P} 1)-(\mathrm{P} 4)$, and $h N \leq C_{3} T$, for an absolute constant $C_{3}$. Then

$$
\|v(\cdot, T)-u(\cdot, T)\|_{L^{1}(\mathbb{R})} \leq C N^{-\min \{\alpha, 1\} / 2}\left|u_{0}\right|_{\operatorname{Lip}\left(1, L^{1}\right)}
$$

where $C$ depends on $M, L, T,\left\|f^{\prime}\right\|_{L^{\infty}(\mathbb{R})}$, (diameter of) the support of $u_{0}$, and $C_{i}$, $0 \leq i \leq 3$.

As a corollary of the theorem, we have the following result for nonoscillatory (NED) schemes.

Corollary 3. Let $u$ be the entropy solution of (21), where $u_{0}$ is a compactly supported function such that $u_{0}$ has at most $K$ local extrema, and let $v$ be the numerical solution obtained by a uniformly bounded nonoscillatory Godunov-type scheme, satisfying (P1)-(P4), and $h N \leq C_{3} T$, for an absolute constant $C_{3}$. Then

$$
\|v(\cdot, T)-u(\cdot, T)\|_{L^{1}(\mathbb{R})} \leq C N^{-\min \{\alpha, 1\} / 2}\left|u_{0}\right|_{\operatorname{Lip}\left(1, L^{1}\right)},
$$

where $C$ depends on $M, K, T,\left\|f^{\prime}\right\|_{L^{\infty}(\mathbb{R})}$, (diameter of) the support of $u_{0}$, and $C_{i}$, $0 \leq i \leq 3$.

\section{WEAKLY NONOSCILLATING FUNCTIONS}

Nonoscillatory schemes play an important role in the theory of conservation laws. A scheme is usually called "nonoscillatory" if it does not increase the number of local extrema of a function. Thus, in particular, if a function is monotone on an interval, then a nonoscillatory method seeks an approximation to the function that preserves this monotonicity.

In the previous section, we generalized the notion of nonoscillation and introduced the class of weakly nonoscillating functions. Roughly, a continuous function $g$ is weakly nonoscillating if the number of intersection points of the graph of $g$ with any horizontal line is at most a given fixed number. This prevents $g$ from oscillating "wildly". It is shown below that this notion of "nonoscillation" is less restrictive than the standard one.

Recall that $\mathcal{W}_{L}$ stands for the class of weakly nonoscillating functions with level sets consisting of at most $L$ components (see Definition [1). The following result shows that this class is closed in $\operatorname{Lip}\left(1, L^{1}\right)$ with respect to the $L^{1}$-topology. 
Theorem 4. Let $L \in \mathbb{N}$ and let $\left\{g_{n}\right\}$ be a sequence of functions in $\mathcal{W}_{L}$ converging to $g \in \operatorname{Lip}\left(1, L^{1}\right)$ in $L^{1}(\mathbb{R})$. Then $g \in \mathcal{W}_{L}$.

Proof. Suppose that $g$ is not in $\mathcal{W}_{L}$. Then there exist values

$$
x_{1}<y_{1}<x_{2}<y_{2}<\cdots<y_{L}<x_{L+1}
$$

such that

$$
\bar{g}\left(x_{i}\right)<\lambda<\bar{g}\left(y_{j}\right), \quad i=1, \ldots, L+1, j=1, \ldots, L,
$$

for some $\lambda \in \mathbb{R}$. Note that $\left\{x_{i}\right\}$ and $\left\{y_{j}\right\}$ can be selected to be points of continuity of $\bar{g}$ (since $\bar{g} \in \mathrm{BV}(\mathbb{R})$ and, hence, has only countably many points of discontinuity). This means we can find an $\varepsilon>0$ such that

$$
\bar{g}(x)<\lambda<\bar{g}(y),
$$

for every $x \in \bigcup_{i=1}^{L+1}\left(x_{i}-\varepsilon, x_{i}+\varepsilon\right)$ and every $y \in \bigcup_{j=1}^{L}\left(y_{j}-\varepsilon, y_{j}+\varepsilon\right)$. However, this and the $L^{1}$ convergence of $\bar{g}_{n}$ to $\bar{g}$ imply that, for all $n$ large enough,

$$
\bar{g}_{n}(x)<\lambda<\bar{g}_{n}(y),
$$

for all $x$ in some sets of positive measure $A_{i} \subset\left(x_{i}-\varepsilon, x_{i}+\varepsilon\right), i=1, \ldots, L+1$, and all $y$ in some sets of positive measure $A_{j}^{*} \subset\left(y_{j}-\varepsilon, y_{j}+\varepsilon\right), j=1, \ldots, L$. Hence, the sets $A_{i}$ belong to different components of $E_{\lambda}\left(g_{n}\right)$. This contradicts the assumption that $g_{n} \in \mathcal{W}_{L}$.

In the remainder of this section, we discuss the relationship between the weakly nonoscillating and the usual nonoscillating functions (i.e., those with finitely many local extrema). First, let us define precisely what it means for a function to have a certain number of extrema. We start by defining extrema for sequences.

Definition 4. Let $\left\{a_{\nu}\right\}_{\nu=1}^{n}, n \in \mathbb{N}$, be a finite sequence of real numbers. We say that this sequence has a strict local maximum (minimum) at some $k(1<k<n)$, if $a_{k}>\max \left\{a_{k-1}, a_{k+1}\right\}\left(a_{k}<\min \left\{a_{k-1}, a_{k+1}\right\}\right)$.

We next define extrema of $\operatorname{Lip}\left(1, L^{1}\right)$ functions in terms of the extrema of their u.s.c. corrections. This reflects the condition that the number of extrema should not change if the functions are modified on sets of measure zero.

Definition 5. A function $g \in \operatorname{Lip}\left(1, L^{1}\right)$ has $K$ local extrema if, for any $x_{1}<x_{2}<$ $\cdots<x_{n}, n \geq 1$, the sequence $\left\{\bar{g}\left(x_{\nu}\right)\right\}_{\nu=1}^{n}$ contains at most $K$ strict local extrema, and $K$ is the smallest integer with this property.

For example, with this definition, the step function does not have any local extrema. The function $g_{1}$ such that $g_{1} \equiv 1$ on $(-\infty, 0]$, and $g_{1}(x)=x, x \in(0, \infty)$ has one local extremum (infimum), and the function $g_{2}$ such that $g_{2}(x)=x+1$, $x \in(-\infty, 0]$, and $g_{2}(x)=x, x \in(0, \infty)$, has two local extrema.

Theorem 5. Let $g \in \operatorname{Lip}\left(1, L^{1}\right)$. If $g$ has $K$ local extrema, then $g \in \mathcal{W}_{L}$, where $L=[(K+3) / 2]$. The converse of this statement is not true for $L \geq 2$ (i.e., a function in $\mathcal{W}_{L}$ can have infinitely many local extrema). In the case $L=1, a$ function from $\mathcal{W}_{1}$ either is monotone or has one local extremum (an infimum). 
Proof. Suppose that $g$ is not in $\mathcal{W}_{L}$. Then, as in the proof of the previous theorem, there exist numbers

$$
x_{1}<y_{1}<x_{2}<y_{2}<\cdots<y_{L}<x_{L+1}
$$

such that

$$
\bar{g}\left(x_{i}\right)<\lambda<\bar{g}\left(y_{j}\right), \quad i=1, \ldots, L+1, j=1, \ldots, L,
$$

for some $\lambda \in \mathbb{R}$. Hence, the sequence

$$
\left\{\bar{g}\left(x_{1}\right), \bar{g}\left(y_{1}\right), \bar{g}\left(x_{2}\right), \bar{g}\left(y_{2}\right), \ldots, \bar{g}\left(y_{L}\right), \bar{g}\left(x_{L+1}\right)\right\}
$$

contains exactly $2 L-1=2[(K+3) / 2]-1>K$ strict local extrema, which is a contradiction.

To show that the converse does not hold, we construct a weakly nonoscillating function with infinitely many local extrema. Let $g_{L}(x):=\sin ((L-1) \pi x) \chi_{[0,2]}(x)$, $x \in \mathbb{R}, L \geq 2$, which is in $\mathcal{W}_{L}$. It is also easy to see that $\left|g_{L}\right|_{\operatorname{Lip}\left(1, L^{1}\right)}=4(L-1)$, and that $g_{L}$ has $2(L-1)$ local extrema in $[0,2]$. Let

$$
g:=\sum_{k=0}^{\infty}\left(1+\frac{1}{4} g_{L}\left(2^{k+2} \cdot-2\right)\right) 2^{-(k+1)} \chi_{\left[2^{-(k+1)}, 2^{-k}\right)} .
$$

This function belongs to $\mathcal{W}_{L}$. In addition, $g$ has $2(L-1)$ local extrema in the interval $\left(2^{-(k+1)}, 2^{-k}\right), k \geq 0$, hence, infinitely many local extrema in $[0,1]$. Also,

$$
\begin{aligned}
|g|_{\operatorname{Lip}\left(1, L^{1}\right)}=|g|_{\mathrm{BV}(\mathbb{R})} & =\frac{1}{2}+\sum_{k=0}^{\infty} 2^{-(k+2)}+\sum_{k=0}^{\infty}|g|_{\mathrm{BV}\left(2^{-(k+1)}, 2^{-k}\right)} \\
& =1+\sum_{k=0}^{\infty} 2^{-(k+3)}\left|g_{L}\right|_{\mathrm{BV}(\mathbb{R})}=L,
\end{aligned}
$$

and so $g \in \operatorname{Lip}\left(1, L^{1}\right)$. The proof of the remaining assertion of the theorem, concerning the case $L=1$, is straightforward.

\section{WEAK NONOSCILLATION OF THE ENTROPY SOLUTION}

In this section, we show that the entropy solution $u(\cdot, t)$ of (2) at any time $t$ is weakly nonoscillating if the initial condition $u_{0}$ is weakly nonoscillating. First, we establish the WNO property of the approximate solution, obtained by the original Godunov method. Then, in Theorem 10, we prove this property for the exact solution, using the convergence of the Godunov method and the completeness of $\mathcal{W}_{L}$ in the $L_{1}$ topology (Theorem 4 ).

Let $T>0$ be fixed and $t_{n}:=n \Delta t, n=0, \ldots, N$, where $\Delta t=T / N$, and define

$$
h:=\Delta t\left(4\left\|f^{\prime}\right\|_{L^{\infty}(\mathbb{R})}+1\right) .
$$

Let us recall the definition of the original Godunov scheme, see e.g., [16, 12]. It is well known that this scheme gives rise to an approximate solution $v:=v^{N}$ that satisfies (7) with $v\left(\cdot, t_{n}\right)=A_{h} v\left(\cdot, t_{n}^{-}\right)$, where $A_{h}$ is the averaging operator, defined in (13). The following error estimate was established in [12] (see also [16]):

$$
\left\|u(\cdot, T)-v^{N}(\cdot, T)\right\|_{L^{1}(\mathbb{R})} \leq \frac{C}{\sqrt{N}}\left|u_{0}\right|_{\operatorname{Lip}\left(1, L^{1}\right)},
$$

where $C$ is a constant independent of $N$. We also need the following result concerning properties of entropy solutions of (2), see [12]. 
Theorem 6. Let $u$ and $w$ be two entropy solutions to (2) with initial conditions $u_{0}$ and $w_{0}$, respectively, and let $f \in \operatorname{Lip}\left(1, L^{\infty}\right)$. Then

$$
\int_{\left|x-x_{0}\right|<R}|u(x, t)-w(x, t)| d x \leq \int_{\left|x-x_{0}\right|<R+t\left\|f^{\prime}\right\|_{L^{\infty}(\mathbb{R})}}\left|u_{0}(x)-w_{0}(x)\right| d x,
$$

for all $t \in[0, T], x_{0} \in \mathbb{R}$, and $R>0$.

Using this estimate, one can easily derive the so-called cone of dependence for the entropy solution.

Corollary 7. Let $t \in[0, T], x_{0} \in \mathbb{R}$, and $R>0$. Then the values of $u(\cdot, t)$ on $\left\{x:\left|x-x_{0}\right|<R\right\}$ depend only on the values of $u_{0}$ on $\left\{x:\left|x-x_{0}\right|<R+t\left\|f^{\prime}\right\|_{L^{\infty}(\mathbb{R})}\right\}$.

There are two repeating steps in the Godunov scheme, the projection (averaging) and the exact evolution. Therefore, it will follow that the numerical solution $v(\cdot, T)$ is in the class $\mathcal{W}_{L}$ if each of the two steps can be shown to preserve this class.

Lemma 8. The averaging operator preserves the class $\mathcal{W}_{L}$. That is, if $g \in \mathcal{W}_{L}$ then $A_{h} g \in \mathcal{W}_{L}$.

Proof. Let $\lambda \in \mathbb{R}$ and let $E_{\lambda}(g)$ be the level set of $g$ corresponding to $\lambda$. Note that $E_{\lambda}(g)$ is empty if $\bar{g} \geq \lambda$. Hence, $g_{j}:=\left.A_{h} g\right|_{I_{j}} \geq \lambda, j \in \mathbb{Z}$, and thus $E_{\lambda}\left(A_{h} g\right)$ is also empty. If $E_{\lambda}(g) \neq \emptyset$, then it is an open set that can be represented as

$$
E_{\lambda}(g)=\bigcup_{\ell=1}^{L_{\lambda}} O_{\ell},
$$

where $O_{\ell}$ are disjoint open intervals and $L_{\lambda} \leq L$. We now show that $E_{\lambda}\left(A_{h} g\right)$ has at most $L_{\lambda}$ components. Observe the following:

(a) If the interior of $I_{j}$ is a subset of $E_{\lambda}(g), \operatorname{int}\left(I_{j}\right) \subset E_{\lambda}(g)$, then $g_{j}<\lambda$ and $\operatorname{int}\left(I_{j}\right) \subset E_{\lambda}\left(A_{h} g\right)$.

(b) If $I_{j} \cap E_{\lambda}(g)=\emptyset$, then $\left.g\right|_{I_{j}} \geq \lambda$; hence $g_{j} \geq \lambda$, and $I_{j} \cap E_{\lambda}\left(A_{h} g\right)=\emptyset$.

Let $J_{\ell}:=\left\{j \mid \operatorname{int}\left(I_{j}\right) \subset E_{\lambda}\left(A_{h} g\right)\right.$ and $\left.I_{j} \cap O_{\ell} \neq \emptyset\right\}$ and

$$
O_{\ell}^{*}:=\operatorname{int}\left(\bigcup_{j \in J_{\ell}} I_{j}\right) .
$$

Note that each $O_{\ell}^{*}$ is either empty or an open interval. This is because by (a), $O_{\ell}^{*}$ consists of all intervals $I_{j}$ contained in $O_{\ell}$ and possibly the end intervals $I_{j}$ such that $I_{j} \cap O_{\ell} \neq \emptyset$ and $I_{j} \not \subset O_{\ell}$ (there could be one or two such end intervals). Moreover, by (b), every nonempty set $O_{\ell}^{*}$ intersects at least one of the sets $O_{k}, k=1, \ldots, L_{\lambda}$. This shows that

$$
E_{\lambda}\left(A_{h} g\right)=\bigcup_{\ell=1}^{L_{\lambda}} O_{\ell}^{*},
$$

and, therefore, $E_{\lambda}\left(A_{h} g\right)$ has at most $L_{\lambda}$ components. Hence, we conclude that $A_{h} g \in \mathcal{W}_{L}$.

Lemma 9. The evolution step does not increase the number of components of the level sets of the numerical solution $v\left(\cdot, t_{n}\right)$. That is, if $v\left(\cdot, t_{n}\right) \in \mathcal{W}_{L}$ is a piecewise constant function on $I_{j}$ 's, then $v\left(\cdot, t_{n+1}^{-}\right) \in \mathcal{W}_{L}, 0 \leq n \leq N-1$. 
Proof. It is enough to prove the assertion for $n=0$. Let $I_{R}\left(x_{0}\right):=\left(x_{0}-R, x_{0}+R\right)$, and $v_{0, j}:=\left.A_{h} u_{0}\right|_{I_{j}}, j \in \mathbb{Z}$. By Corollary 7 , for any $x_{0} \in \mathbb{R}$, the values of $v(\cdot, \Delta t)$ on $I_{R}\left(x_{0}\right)$ are determined by the values of $A_{h} u_{0}$ on $I_{R+\left\|f^{\prime}\right\|_{L \infty(\mathbb{R})} \Delta t}\left(x_{0}\right)$. Note that $\left\|f^{\prime}\right\|_{L^{\infty}(\mathbb{R})} \Delta t \leq h / 4$. Therefore, for any integer $j \in \mathbb{Z}, v(\cdot, \Delta t)$ and the entropy solution of the Riemann problem

$$
\left\{\begin{array}{l}
u_{t}+f(u)_{x}=0, \\
u(x, 0)=\left\{\begin{array}{ll}
v_{0, j-1}, & x<j h, \\
v_{0, j}, & x>j h,
\end{array} \quad(x, t) \in \mathbb{R} \times(0, \Delta t),\right.
\end{array}\right.
$$

are identical on the interval $I_{h / 2}(j h)$. Note that

$$
v((j+1 / 2) h+\varepsilon, \Delta t)=v_{0, j},
$$

for any $\varepsilon,|\varepsilon|<h / 4$, and the function $v(\cdot, \Delta t)$ is monotone on $I_{h / 2}(j h)$ (see (44)). Hence, $v(\cdot, \Delta t)$ takes on the values $v_{0, j}, j \in \mathbb{Z}$, in the same order as $A_{h} u_{0}$ and is monotone in between. Then, for any $\lambda \in \mathbb{R}$, the level set $E_{\lambda}(v(\cdot, \Delta t))$ has the same number of components as $E_{\lambda}\left(A_{h} u_{0}\right)$. Therefore, we conclude that $v(\cdot, \Delta t) \in \mathcal{W}_{L}$ if $A_{h} u_{0} \in \mathcal{W}_{L}$

We now conclude with the main result of this section.

Theorem 10. Let $u$ be the entropy solution of (2) with compactly supported initial condition $u_{0} \in \operatorname{Lip}\left(1, L^{1}\right)$. If $u_{0} \in \mathcal{W}_{L}$, for some $L \in \mathbb{N}$, then $u(\cdot, t) \in \mathcal{W}_{L}$, $t \in[0, T]$.

Proof. Let $L \in \mathbb{N}$ and $u_{0} \in \mathcal{W}_{L}$. Since $T$ can be an arbitrary positive number, it is sufficient to show that $u(\cdot, T) \in \mathcal{W}_{L}$. Using Lemmas 8 and 9 we see that $v^{N}(\cdot, T) \in \mathcal{W}_{L}$. By the estimate (19), we know that $v^{N}(\cdot, T)$ converges to $u(\cdot, T)$ in $L^{1}(\mathbb{R})$. Hence, we can apply Theorem 4 to $v^{N}(\cdot, T)$ and $u(\cdot, T)$, to conclude that $u(\cdot, T) \in \mathcal{W}_{L}$.

\section{Proof of the main Result}

The proof of Theorem 2 will require a judicious estimation of the sum on the right-hand side of the Kuznetsov inequality (8). First, we introduce the notation $v_{n}^{-}:=v\left(\cdot, t_{n}^{-}\right), v_{n}:=v\left(\cdot, t_{n}\right)=P_{h} v_{n}^{-}, u_{n}:=u\left(\cdot, t_{n}\right)$ (recall that $\left.v_{0}^{-}=u_{0}\right)$. Estimate (8) can now be rewritten as

$$
\left\|v_{N}-u_{N}\right\|_{L^{1}(\mathbb{R})} \leq\left\|v_{0}-u_{0}\right\|_{L^{1}(\mathbb{R})}+2 \varepsilon\left|u_{0}\right|_{B V(\mathbb{R})}+\sum_{n=1}^{N}\left[\rho_{\varepsilon}\left(v_{n}, u_{n}\right)-\rho_{\varepsilon}\left(v_{n}^{-}, u_{n}\right)\right] .
$$

Let us denote

$$
\Delta_{n}:=\rho_{\varepsilon}\left(v_{n}, u_{n}\right)-\rho-\varepsilon\left(v_{n}^{-}, u_{n}\right)
$$

and

$$
F_{n}(x, y):=\left|P_{h} v_{n}^{-}(x)-u_{n}(y)\right|-\left|v_{n}^{-}(x)-u_{n}(y)\right|,
$$


and choose $\eta:=\frac{1}{2} \chi_{[-1,1]}$. Then

$$
\begin{aligned}
\Delta_{n} & =\int_{\mathbb{R}} \int_{\mathbb{R}} \eta_{\varepsilon}(x-y) F_{n}(x, y) d x d y \\
& =\frac{1}{2 \varepsilon} \int_{\mathbb{R}} \int_{\mathbb{R}} \chi_{[-1,1]}\left(\frac{x-y}{\varepsilon}\right) F_{n}(x, y) d x d y \\
& =\frac{1}{2 \varepsilon} \int_{\mathbb{R}}\left(\int_{[y-\varepsilon, y+\varepsilon]} F_{n}(x, y) d x\right) d y \\
& =\frac{1}{2 \varepsilon} \int_{\mathbb{R}}\left(\sum_{j \in \mathbb{Z}} \int_{I_{j} \cap[y-\varepsilon, y+\varepsilon]} F_{n}(x, y) d x\right) d y .
\end{aligned}
$$

The above sum can now be split into two sums, depending on whether $I_{j}$ is contained in $(y-\varepsilon, y+\varepsilon)$ or not:

$$
\begin{aligned}
\Delta_{n} & =\frac{1}{2 \varepsilon} \int_{\mathbb{R}} \sum_{I_{j} \cap\{y-\varepsilon, y+\varepsilon\} \neq \emptyset} \int_{I_{j} \cap[y-\varepsilon, y+\varepsilon]} F_{n}(x, y) d x d y \\
& +\frac{1}{2 \varepsilon} \int_{\mathbb{R}_{I_{j}}} \sum_{(y-\varepsilon, y+\varepsilon)} \int_{I_{j}} F_{n}(x, y) d x d y=\Delta_{n}^{1}+\Delta_{n}^{2},
\end{aligned}
$$

where

$$
\Delta_{n}^{1}:=\frac{1}{2 \varepsilon} \int_{\mathbb{R}} \sum_{I_{j} \cap\{y-\varepsilon, y+\varepsilon\} \neq \emptyset} \int_{I_{j} \cap[y-\varepsilon, y+\varepsilon]} F_{n}(x, y) d x d y
$$

and

$$
\Delta_{n}^{2}:=\frac{1}{2 \varepsilon} \int_{\mathbb{R}} \sum_{I_{j} \subset(y-\varepsilon, y+\varepsilon)} \int_{I_{j}} F_{n}(x, y) d x d y .
$$

Using the triangle inequality $F_{n}(x, y) \leq\left|P_{h} v_{n}^{-}(x)-v_{n}^{-}(x)\right|$, we estimate $\Delta_{n}^{1}$ as follows:

$$
\begin{aligned}
\Delta_{n}^{1} & \leq \frac{1}{2 \varepsilon} \int_{\mathbb{R}} \sum_{I_{j} \cap\{y-\varepsilon, y+\varepsilon\} \neq \emptyset} \int_{I_{j}}\left|P_{h} v_{n}^{-}(x)-v_{n}^{-}(x)\right| d x d y \\
& =\frac{1}{2 \varepsilon} \int_{\mathbb{R}_{I_{j} \cap\{y-\varepsilon, y+\varepsilon\} \neq \emptyset}} \sum_{\left\|P_{h} v_{n}^{-}-v_{n}^{-}\right\|_{L^{1}\left(I_{j}\right)} d y .}
\end{aligned}
$$

Now, the following fact is useful.

For any real sequence $\left\{a_{j}\right\}_{j \in \mathbb{Z}}$, and $\lambda \in \mathbb{R}$,

$$
\begin{aligned}
\int_{\mathbb{R}} & \left(\sum_{I_{j} \cap\{y+\lambda\} \neq \emptyset} a_{j}\right) d y \\
= & \int_{\mathbb{R}}\left(\sum_{I_{j} \cap\{y\} \neq \emptyset} a_{j}\right) d y=\sum_{k \in \mathbb{Z}} \int_{I_{k}}\left(\sum_{I_{j} \cap\{y\} \neq \emptyset} a_{j}\right) d y \\
= & \sum_{k \in \mathbb{Z}} \int_{I_{k}} a_{k} d y=\sum_{k \in \mathbb{Z}} a_{k} \operatorname{meas}\left(I_{k}\right) .
\end{aligned}
$$


Using (22) with $a_{j}=\left\|P_{h} v_{n}^{-}-v_{n}^{-}\right\|_{L^{1}\left(I_{j}\right)}$ and $\lambda= \pm \varepsilon$, we have

$$
\begin{aligned}
\Delta_{n}^{1} & \leq \frac{1}{2 \varepsilon} \sum_{k \in \mathbb{Z}} 2\left\|P_{h} v_{n}^{-}-v_{n}^{-}\right\|_{L^{1}\left(I_{k}\right)} \operatorname{meas}\left(I_{k}\right)=\frac{h}{\varepsilon} \sum_{k \in \mathbb{Z}}\left\|P_{h} v_{n}^{-}-v_{n}^{-}\right\|_{L^{1}\left(I_{k}\right)} \\
& =\frac{h}{\varepsilon}\left\|P_{h} v_{n}^{-}-v_{n}^{-}\right\|_{L^{1}(\mathbb{R})} .
\end{aligned}
$$

By the approximation property (12),

$$
\left\|P_{h} v_{n}^{-}-v_{n}^{-}\right\|_{L^{1}(\mathbb{R})} \leq\left(1+C_{0}\right) h\left|v_{n}^{-}\right|_{\operatorname{Lip}\left(1, L^{1}\right)},
$$

and, therefore,

$$
\Delta_{n}^{1} \leq\left(1+C_{0}\right) \frac{h^{2}}{\varepsilon}\left|v_{n}^{-}\right|_{\operatorname{Lip}\left(1, L^{1}\right)} .
$$

We now estimate $\Delta_{n}^{2}$. Note that if $\varepsilon<h / 2$, then $I_{j}$ cannot be contained in $(y-\varepsilon, y+\varepsilon)$, and hence $\Delta_{n}^{2}=0$. So, in the remainder of the proof, we assume $\varepsilon \geq h / 2$.

Let us define

$$
\left.\Omega_{n}:=\bigcup\left\{I_{j} \mid I_{j} \cap \operatorname{supp}\left(P_{h} v_{n}^{-}\right) \neq \emptyset \text { or } I_{j} \cap \operatorname{supp}\left(v_{n}^{-}\right)\right) \neq \emptyset\right\} .
$$

Then if $x \notin \Omega_{n}$, we have $P_{h} v_{n}^{-}(x)=v_{n}^{-}(x)=0$. Therefore, $F_{n}(x, y)=0$ for all $x \notin \Omega_{n}$. Hence, if $(y-\varepsilon, y+\varepsilon) \cap \Omega_{n}=\emptyset$, then

$$
\sum_{I_{j} \subset(y-\varepsilon, y+\varepsilon)} \int_{I_{j}} F_{n}(x, y) d x=0,
$$

which yields the estimate

$$
\Delta_{n}^{2} \leq \frac{1}{2 \varepsilon} \int_{\Omega_{n}^{\varepsilon}}\left(\sum_{I_{j} \subset(y-\varepsilon, y+\varepsilon)} \int_{I_{j}} F_{n}(x, y) d x\right) d y,
$$

where $\Omega_{n}^{\varepsilon}:=\left\{y \mid(y-\varepsilon, y+\varepsilon) \cap \Omega_{n} \neq \emptyset\right\}$. We next need the following lemma.

Lemma 11. Let $g \in \mathcal{W}_{L}, L \geq 1$. Then, for any $\lambda \in \mathbb{R}$, there exist at most $2 L$ intervals $I_{j}$ such that

$$
\text { meas }\left\{x \in I_{j} \mid g(x)>\lambda\right\}>0
$$

and

$$
\text { meas }\left\{x \in I_{j} \mid g(x)<\lambda\right\}>0
$$

at the same time.

For a given $\lambda$, we denote the set of all such indices $j$ by $V_{\lambda}(g)$ (hence, the lemma states that $\left|V_{\lambda}(g)\right| \leq 2 L$, for any $\lambda \in \mathbb{R}$, where $|A|$ stands for the cardinality of the set $A)$.

Proof. Suppose that $V_{\lambda}(g)$ is nonempty and ordered as $V_{\lambda}(g)=\left\{j_{k}\right\}_{k=1}^{\left|V_{\lambda}(g)\right|}$, where $j_{1}<\cdots<j_{\left|V_{\lambda}(g)\right|}$. Since $g \in \mathcal{W}_{L}, E_{\lambda}:=E_{\lambda}(g)$ has at most $L$ components, i.e.,

$$
E_{\lambda}=\bigcup_{\ell=1}^{L_{\lambda}} O_{\ell}, \quad L_{\lambda} \leq L,
$$

where all $O_{\ell}$ are disjoint open intervals. For each $j \in V_{\lambda}(g)$,

$$
\text { meas }\left\{I_{j} \cap E_{\lambda}\right\}>0 \quad \text { (by the definition of } V_{\lambda}(g) \text { ) }
$$


and

$$
\text { meas }\left\{I_{j} \cap E_{\lambda}^{c}\right\}>0 \quad\left(\begin{array}{c}
\text { since } \left.\underset{I_{j}}{\operatorname{essup}} g>\lambda\right) . \\
V_{\lambda}(g), \text { ther }
\end{array}\right.
$$

Inequality (24) implies that, for every $j \in V_{\lambda}(g)$, there is at least one $\ell(j), 1 \leq$ $\ell(j) \leq L_{\lambda}$, such that $I_{j} \cap O_{\ell(j)} \neq \emptyset$. Note that if there are more than one $\ell(j)$, then we select any one of them. Now, (25) implies that $I_{j} \not \subset E_{\lambda}, j \in V_{\lambda}(g)$. This, in particular, means that $\ell\left(j_{k}\right) \neq \ell\left(j_{k+2}\right)$ for all $k=1, \ldots,\left|V_{\lambda}(g)\right|-2$; otherwise $I_{j_{k+1}}$ would be contained in $O_{\ell\left(j_{k}\right)}$, which contradicts (25)). Therefore,

$$
\left|V_{\lambda}(g)\right| \leq 2\left|\left\{\ell(j) \mid j \in V_{\lambda}(g)\right\}\right| \leq 2 L_{\lambda} \leq 2 L .
$$

Let $y \in \Omega_{n}^{\varepsilon}$ be fixed and $\lambda:=u_{n}(y)$. Because $P_{h}$ is conservative, the expressions $P_{h} v_{n}^{-}(x)-\lambda$ and $v_{n}^{-}(x)-\lambda$ have the same sign on $I_{j}$ whenever $j \notin J_{\lambda}:=V_{\lambda}\left(P_{h} v_{n}^{-}\right) \cup$ $V_{\lambda}\left(v_{n}^{-}\right)$. Hence, $F_{n}(x, y)= \pm\left(P_{h} v_{n}^{-}(x)-v_{n}^{-}(x)\right)$ and, therefore, $\int_{I_{j}} F_{n}(x, y) d x=0$ for $j \notin J_{\lambda}$.

Now, let $j \in J_{\lambda}$. Then, by (11),

$$
\int_{I_{j}} F_{n}(x, y) d x \leq C_{1} h^{1+\alpha}\left|v_{n}^{-}\right|_{\operatorname{Lip}\left(1, L^{1}\right)} .
$$

Hence, using Lemma 11 and the fact that $P_{h} v_{n}^{-} \in \mathcal{W}_{L}$, we obtain

$$
\begin{aligned}
\Delta_{n}^{2} & \leq \frac{1}{2 \varepsilon} \int_{\Omega_{n}^{\varepsilon}}\left(\sum_{j \in J_{u_{n}(y)}} C_{1} h^{1+\alpha}\left|v_{n}^{-}\right|_{\operatorname{Lip}\left(1, L^{1}\right)}\right) d y \\
& =\frac{C_{1}}{2 \varepsilon} h^{1+\alpha}\left|v_{n}^{-}\right|_{\operatorname{Lip}\left(1, L^{1}\right)} \int_{\Omega_{n}^{\varepsilon}}\left|J_{u_{n}(y)}\right| d y \\
& \leq \frac{2 C_{1} L}{\varepsilon} \operatorname{meas}\left(\Omega_{n}^{\varepsilon}\right) h^{1+\alpha}\left|v_{n}^{-}\right|_{\operatorname{Lip}\left(1, L^{1}\right)} .
\end{aligned}
$$

We now estimate meas $\left(\Omega_{n}^{\varepsilon}\right)$. Recall that $\operatorname{diam}(A), A \subset \mathbb{R}$, is the length of the smallest interval containing $A$. We have

$$
\begin{aligned}
\operatorname{meas}\left(\Omega_{n}^{\varepsilon}\right) & \leq \operatorname{diam}\left(\Omega_{n}^{\varepsilon}\right) \leq 2 \varepsilon+\operatorname{diam}\left(\Omega_{n}\right) \\
& \leq 2 \varepsilon+2 h+\operatorname{diam}\left(\operatorname{supp}\left(P_{h} v_{n}^{-}\right) \cup \operatorname{supp}\left(v_{n}^{-}\right)\right) \\
& \leq 2 \varepsilon+2 h+2(M+1) h+\operatorname{diam}\left(\operatorname{supp}\left(v_{n}^{-}\right)\right),
\end{aligned}
$$

where we used the locality of $P_{h}$ (property (P3)).

To estimate $\operatorname{diam}\left(\operatorname{supp}\left(v_{n}^{-}\right)\right)$we need the following two inequalities:

$$
\operatorname{diam}\left(\operatorname{supp}\left(v_{n}^{-}\right)\right) \leq \operatorname{diam}\left(\operatorname{supp}\left(v_{n-1}\right)\right)+2\left\|f^{\prime}\right\|_{L^{\infty}(\mathbb{R})}\left(t_{n}-t_{n-1}\right),
$$

which follows from Corollary 7 and

$$
\operatorname{diam}\left(\operatorname{supp}\left(v_{n-1}\right)\right)=\operatorname{diam}\left(\operatorname{supp}\left(P_{h} v_{n-1}^{-}\right)\right) \leq \operatorname{diam}\left(\operatorname{supp}\left(v_{n-1}^{-}\right)\right)+2(M+1) h .
$$

Combining these inequalities for $k=0, \ldots, n$, we have

$$
\begin{aligned}
& \operatorname{diam}\left(\operatorname{supp}\left(v_{n}^{-}\right)\right) \\
& \quad \leq 2\left\|f^{\prime}\right\|_{L^{\infty}(\mathbb{R})} \sum_{k=0}^{n-1}\left(t_{k+1}-t_{k}\right)+2 \sum_{k=0}^{n-1}(M+1) h+\operatorname{diam}\left(\operatorname{supp}\left(u_{0}\right)\right) \\
& =2 t_{n}\left\|f^{\prime}\right\|_{L^{\infty}(\mathbb{R})}+2(M+1) h n+\operatorname{diam}\left(\operatorname{supp}\left(u_{0}\right)\right) .
\end{aligned}
$$


Hence, for all $1 \leq n \leq N$, we have

$$
\operatorname{diam}\left(\operatorname{supp}\left(v_{n}^{-}\right)\right) \leq 2 T\left\|f^{\prime}\right\|_{L^{\infty}(\mathbb{R})}+2(M+1) h N+\operatorname{diam}\left(\operatorname{supp}\left(u_{0}\right)\right) .
$$

Consequently,

$$
\begin{aligned}
\Delta_{n}^{2} \leq \frac{2 C_{1} L}{\varepsilon} \quad & {\left[2 \varepsilon+2 h+2(M+1) h(N+1)+2 T\left\|f^{\prime}\right\|_{L^{\infty}(\mathbb{R})}\right.} \\
& \left.+\operatorname{diam}\left(\operatorname{supp}\left(u_{0}\right)\right)\right] h^{1+\alpha}\left|v_{n}^{-}\right|_{\operatorname{Lip}\left(1, L^{1}\right)}
\end{aligned}
$$

or

$$
\Delta_{n}^{2} \leq \frac{C_{1} C_{4} L}{\varepsilon} h^{1+\alpha}\left|v_{n}^{-}\right|_{\operatorname{Lip}\left(1, L^{1}\right)},
$$

where

$$
C_{4}:=2\left[2+2(2 M+3) C_{3} T+2 T\left\|f^{\prime}\right\|_{L^{\infty}(\mathbb{R})}+\operatorname{diam}\left(\operatorname{supp}\left(u_{0}\right)\right)\right]
$$

(note that we assumed that $\varepsilon \leq 1$ and used $h \leq 2 \varepsilon \leq 2$ ).

We are now ready to finish the proof of Theorem 2, First, combining (23) and (26), we obtain

$$
\begin{aligned}
\sum_{n=1}^{N} \Delta_{n} & =\sum_{n=1}^{N} \Delta_{n}^{1}+\sum_{n=1}^{N} \Delta_{n}^{2} \\
& \leq\left(\left(1+C_{0}\right) \frac{h^{2}}{\varepsilon}+L C_{1} C_{4} \frac{h^{1+\alpha}}{\varepsilon}\right) \sum_{n=1}^{N}\left|v_{n}^{-}\right|_{\operatorname{Lip}\left(1, L^{1}\right)} . \\
& \leq\left(1+C_{0}+L C_{1} C_{4}\right) \frac{h^{1+\min \{\alpha, 1\}}}{\varepsilon} \sum_{n=1}^{N}\left|v_{n}^{-}\right|_{\operatorname{Lip}\left(1, L^{1}\right)} .
\end{aligned}
$$

Next, we use the fact that

$$
\begin{aligned}
\left|v_{n}^{-}\right|_{\operatorname{Lip}\left(1, L^{1}\right)} & \leq\left|v_{n-1}\right|_{\operatorname{Lip}\left(1, L^{1}\right)} \leq 4 L\left\|v_{n-1}\right\|_{L^{\infty}(\mathbb{R})} \\
& \leq 4 L C_{2}\left\|u_{0}\right\|_{L^{\infty}(\mathbb{R})} \leq 4 L C_{2}\left\|u_{0}\right\|_{\operatorname{Lip}\left(1, L^{1}\right)},
\end{aligned}
$$

where the first inequality follows from the fact that the entropy solution is variation diminishing (see (4)), the second inequality is a consequence of (14), the third estimate follows from the fact that the scheme is uniformly bounded (see Definition 3 ), while the last estimate follows from (15). Hence,

$$
\sum_{n=1}^{N} \Delta_{n} \leq 4 L C_{2}\left(1+C_{0}+L C_{1} C_{4}\right) \frac{h^{1+\min \{\alpha, 1\}} N}{\varepsilon}\left|u_{0}\right|_{\operatorname{Lip}\left(1, L^{1}\right)} .
$$

Thus, recalling inequality (8),

$$
\begin{aligned}
\| v_{N} & -u_{N}\left\|_{L^{1}(\mathbb{R})} \leq\right\| u_{0}-v_{0} \|_{L^{1}(\mathbb{R})} \\
& +\left|u_{0}\right|_{\operatorname{Lip}\left(1, L^{1}\right)}\left[2 \varepsilon+4 L C_{2}\left(1+C_{0}+L C_{1} C_{4}\right) \frac{h^{1+\min \{\alpha, 1\}} N}{\varepsilon}\right] .
\end{aligned}
$$

Now, choosing $\varepsilon:=N^{-\min \{\alpha, 1\} / 2}$ and recalling that $h N \leq C_{3} T$, we get

$$
\left\|u_{N}-v_{N}\right\|_{L^{1}(\mathbb{R})} \leq\left\|u_{0}-v_{0}\right\|_{L^{1}(\mathbb{R})}+C_{5} N^{-\min \{\alpha, 1\} / 2}\left|u_{0}\right|_{\operatorname{Lip}\left(1, L^{1}\right)},
$$

where

$$
C_{5}:=\left[2+4 L C_{2}\left(1+C_{0}+L C_{1} C_{4}\right)\left(C_{3} T\right)^{1+\min \{\alpha, 1\}}\right] .
$$


Using (12), we now estimate the first term:

$$
\begin{aligned}
\left\|u_{0}-v_{0}\right\|_{L^{1}(\mathbb{R})} & =\left\|P_{h} u_{0}-u_{0}\right\|_{L^{1}(\mathbb{R})} \leq\left(1+C_{0}\right) h\left|u_{0}\right|_{\operatorname{Lip}\left(1, L^{1}\right)} \\
& \leq \frac{\left(1+C_{0}\right) C_{3} T}{N}\left|u_{0}\right|_{\operatorname{Lip}\left(1, L^{1}\right)} .
\end{aligned}
$$

Therefore, the final estimate is

$$
\left\|u_{N}-v_{N}\right\|_{L^{1}(\mathbb{R})} \leq C N^{-\min \{\alpha, 1\} / 2}\left|u_{0}\right|_{\operatorname{Lip}\left(1, L^{1}\right)},
$$

where $C$ depends on $L, M, T,\left\|f^{\prime}\right\|_{L^{\infty}(\mathbb{R})}, \operatorname{diam}\left(\operatorname{supp}\left(u_{0}\right)\right)$, and $C_{i}, 0 \leq i \leq 3$.

\section{EXAmples}

In this section, we give some examples of relaxed entropic schemes (see (E1)(E3)). We also give an example (E4) of a scheme that is not relaxed entropic, satisfies all other conditions of our main result (Theorem 2), and is convergent to a weak solution which is different from the entropy solution. This shows that the condition that the scheme is relaxed entropic cannot be removed.

Recall that we consider schemes with exact evolution, i.e., we assume that we can solve the conservation law (7) exactly for all time intervals $\left(t_{n}, t_{n+1}\right)$, where $v\left(\cdot, t_{n}\right)=P_{h} v\left(\cdot, t_{n}^{-}\right), 0 \leq n \leq N-1$. Here, we restrict ourselves to the special case where $P_{h}$ is a conservative projection onto piecewise linear functions. That is, for $g \in L^{1}(\mathbb{R})$,

$$
P_{h} g(x):=g_{j}+\sigma_{j}(x-(j+1 / 2) h), \quad x \in I_{j}, \quad j \in \mathbb{Z},
$$

where $g_{j}:=\frac{1}{h} \int_{I_{j}} g d x$ and $\sigma_{j}$ are appropriately chosen slopes. We also recall the definition of the classical MinMod limiter

$$
\mu(a, b):= \begin{cases}\operatorname{sgn}(a) \min (|a|,|b|), & a b>0 \\ 0, & a b \leq 0\end{cases}
$$

and its extension

$$
\mu(A):= \begin{cases}\inf (A), & A \subset \mathbb{R}_{+} \\ \sup (A), & A \subset \mathbb{R}_{-} \\ 0, & \text { otherwise }\end{cases}
$$

We now describe examples of nonoscillatory schemes corresponding to different choices of $\sigma_{j}$ in (30). Observe that such schemes are WNO with constant $L=$ $[(K+3) / 2]$, where $K$ is the number of local extrema of $u_{0}$ (see Remark 7 and also Theorem [5).

(E1) Entropic scheme (Bouchut et al. [1]):

$$
\sigma_{j}:=\mu\left(\zeta_{j}(y), y \in I_{j}\right), \quad j \in \mathbb{Z},
$$

where

$$
\zeta_{j}(y):=\frac{2}{h}\left(\frac{1}{(j+1) h-y} \int_{y}^{(j+1) h} g d x-\frac{1}{y-j h} \int_{j h}^{y} g d x\right) .
$$

This scheme is total variation diminishing and entropic (see Proposition 3.4 in [1]). Thus, the projection $P_{h}$ in this case satisfies properties (P1)-(P4) with $C_{0}=C_{1}=0$. 


\section{(E2) Modified MinMod:}

$$
\sigma_{j}:=\operatorname{sgn}\left(\sigma_{j}^{\prime}\right) \min \left(\left|\sigma_{j}^{\prime}\right|, C h^{\alpha^{\prime}-1} \sum_{\nu=j-J-1}^{j+J}\left|\Delta g_{\nu}\right|\right),
$$

where $J \geq 0$ is a fixed integer,

$$
\sigma_{j}^{\prime}:=\frac{1}{h} \mu\left(\Delta g_{j-1}, \Delta g_{j}\right)
$$

$\Delta g_{\nu}:=g_{\nu+1}-g_{\nu}, C$ is an absolute constant, and $\alpha^{\prime}>0$. Note that $P_{h}$ in (30), with $\sigma_{j}$ replaced by $\sigma_{j}^{\prime}$, is the original MinMod projection. Alternatively, the classical MinMod scheme (with exact evolution) can be thought of as a limiting case of this modified scheme if $\alpha^{\prime} \rightarrow 0$ and $C \geq 1 / 2$. However, we cannot set $\alpha^{\prime}$ equal to zero, since then Theorem 2 does not yield convergence. In fact, while there is numerical evidence suggesting that the original MinMod is convergent, to our best knowledge this has not yet been proved rigorously.

Observe that the usual MinMod projection is not entropic (not even relaxed entropic). On the other hand, we show next that the modified projection is relaxed entropic. Using the triangle inequality, we have, for $g \in \operatorname{Lip}\left(1, L^{1}\right)$,

$$
\begin{aligned}
\int_{I_{j}} & \left(\left|P_{h} g(x)-\lambda\right|-|g(x)-\lambda|\right) d x \\
& \leq \int_{I_{j}}\left|P_{h} g(x)-A_{h} g(x)\right| d x+\int_{I_{j}}\left(\left|A_{h} g(x)-\lambda\right|-|g(x)-\lambda|\right) d x,
\end{aligned}
$$

where $A_{h}$ is the averaging operator defined in (13). Since $A_{h}$ is entropic, the second integral on the right-hand side is nonpositive; hence

$$
\begin{aligned}
& \int_{I_{j}}\left(\left|P_{h} g(x)-\lambda\right|-|g(x)-\lambda|\right) d x \\
& \quad \leq \int_{I_{j}}\left|P_{h} g(x)-A_{h} g(x)\right| d x=\frac{\left|\sigma_{j}\right| h^{2}}{4} \\
& \leq \frac{C}{4} h^{1+\alpha^{\prime}} \sum_{\nu=j-J-1}^{j+J}\left|\Delta g_{\nu}\right| \leq \frac{C}{4} h^{1+\alpha^{\prime}}\left|A_{h} g\right|_{\mathrm{BV}(\mathbb{R})} \\
& \quad \leq \frac{C}{4} h^{1+\alpha^{\prime}}|g|_{\operatorname{Lip}\left(1, L^{1}\right)},
\end{aligned}
$$

which shows that (P4) holds with $C_{1}=C / 4$ and $\alpha=\alpha^{\prime}$ in (11). The remaining properties (P1)-(P3) are satisfied with $C_{0}=0$ and $M=J+1$, since it is well known that the original MinMod method is total variation diminishing and is obviously local.

(E3) Modified UNO:

$$
\sigma_{j}:=\operatorname{sgn}\left(\sigma_{j}^{\prime \prime}\right) \min \left(\left|\sigma_{j}^{\prime \prime}\right|, C \sum_{\nu=j-J-1}^{j+J}\left|\Delta g_{\nu}\right|\right),
$$

where $J \geq 0, C$ is an absolute constant, and

$$
\sigma_{j}^{\prime \prime}:=\frac{1}{h} \mu\left(\Delta g_{j-1}+\frac{1}{2} \mu\left(\Delta^{2} g_{j-1}, \Delta^{2} g_{j}\right), \Delta g_{j}-\frac{1}{2} \mu\left(\Delta^{2} g_{j}, \Delta^{2} g_{j+1}\right)\right),
$$


where $\Delta^{2} g_{\nu}:=\Delta\left(\Delta g_{\nu}\right)=g_{\nu+2}-2 g_{\nu+1}+g_{\nu}$. Note that $\sigma_{j}^{\prime \prime}$ corresponds to the slope in the original UNO method introduced in [7. In the above expression for $\sigma_{j}$, we use an absolute constant $C$, rather than $C h^{\alpha^{\prime}-1}$, as in example (E2), because this will guarantee property (P2). This can be shown as follows:

$$
\begin{aligned}
\left|P_{h} g\right|_{\mathrm{Lip}\left(1, L^{1}\right)} & =\sum_{j \in \mathbb{Z}}\left|P_{h} g\right|_{\mathrm{BV}(j h,(j+1) h)}+\sum_{j \in \mathbb{Z}}\left|P_{h} g\left((j h)^{+}\right)-P_{h} g\left((j h)^{-}\right)\right| \\
& \leq \sum_{j \in \mathbb{Z}}\left|P_{h} g\right|_{\mathrm{BV}(j h,(j+1) h)}+\sum_{j \in \mathbb{Z}}\left|\Delta g_{j}\right|+\sum_{j \in \mathbb{Z}} \frac{h}{2}\left(\left|\sigma_{j-1}\right|+\left|\sigma_{j}\right|\right) \\
& =\left|A_{h} g\right|_{\mathrm{BV}(\mathbb{R})}+2 h \sum_{j \in \mathbb{Z}}\left|\sigma_{j}\right|,
\end{aligned}
$$

since $\sum_{j \in \mathbb{Z}}\left|\Delta g_{j}\right|=\left|A_{h} g\right|_{\mathrm{BV}(\mathbb{R})}$ and $\left|P_{h} g\right|_{\mathrm{BV}(j h,(j+1) h)}=h\left|\sigma_{j}\right|$. Using the inequality $\left|\sigma_{j}\right| \leq C \sum_{\nu=j-J-1}^{j+J}\left|\Delta g_{\nu}\right|$, we arrive at

$$
\begin{aligned}
\left|P_{h} g\right|_{\operatorname{Lip}\left(1, L^{1}\right)} & \leq\left|A_{h} g\right|_{\mathrm{BV}(\mathbb{R})}+2 C h \sum_{j \in \mathbb{Z}} \sum_{\nu=j-J-1}^{j+J}\left|\Delta g_{\nu}\right| \\
& =\left|A_{h} g\right|_{\mathrm{BV}(\mathbb{R})}+4 C(J+1) h \sum_{j \in \mathbb{Z}}\left|\Delta g_{j}\right| \\
& =(1+4 C(J+1) h)\left|A_{h} g\right|_{\mathrm{BV}(\mathbb{R})} \leq(1+4 C(J+1) h)|g|_{\operatorname{Lip}\left(1, L^{1}\right)}
\end{aligned}
$$

which proves property (P2) with $C_{0}=1+\tilde{C} h:=1+4 C(J+1) h$. This estimate guarantees that the method is uniformly bounded. Indeed, using (4), we have

$$
\left|v_{n}\right|_{\operatorname{Lip}\left(1, L^{1}\right)}=\left|P_{h} v_{n}^{-}\right|_{\operatorname{Lip}\left(1, L^{1}\right)} \leq(1+\tilde{C} h)\left|v_{n}^{-}\right|_{\operatorname{Lip}\left(1, L^{1}\right)} \leq(1+\tilde{C} h)\left|v_{n-1}\right|_{\operatorname{Lip}\left(1, L^{1}\right)} .
$$

Hence, using (15) and $h N \leq C_{3} T$, we conclude that

$$
\begin{aligned}
\left\|v_{n}\right\|_{L^{\infty}(\mathbb{R})} & \leq\left|v_{n}\right|_{\operatorname{Lip}\left(1, L^{1}\right)} \leq(1+\tilde{C} h)^{n+1}\left|u_{0}\right|_{\operatorname{Lip}\left(1, L^{1}\right)} \\
& \leq\left(1+\tilde{C} C_{3} T / N\right)^{N+1}\left|u_{0}\right|_{\operatorname{Lip}\left(1, L^{1}\right)} \leq C\left\|u_{0}\right\|_{L^{\infty}(\mathbb{R})} .
\end{aligned}
$$

Note that the total variation of the numerical solution obtained by the original UNO method may still be uniformly bounded for all time steps. However, even though this is observed experimentally, we are not aware of a rigorous proof of this fact.

Finally, (P1) and (P3) are also satisfied, where, for the latter, we have $M=$ $\max \{2, J+1\}$, and (P4) holds with $\alpha=1$ (this can be proved as in the previous example).

To summarize, in the above examples, the projections $P_{h}$ satisfy conditions (P1)(P4) and the corresponding schemes are WNO (for nonoscillating initial data). Hence, if the initial condition $u_{0} \in \operatorname{Lip}\left(1, L^{1}\right)$ is compactly supported and has finitely many extrema, Theorem 2 can be applied to establish the convergence of these schemes to the entropy solution of (2). Moreover, the estimate (16) is satisfied with $\alpha=1, \alpha^{\prime}, 1$ for the schemes (E1), (E2), and (E3), respectively.

\section{(E4) A counterexample}

In the remainder of this section, we construct an example of a nonoscillatory scheme which is not relaxed entropic, satisfies all other assumptions of Theorem 2, but is not convergent to the entropy solution. This shows that one cannot simply discard the relaxed entropy assumption (P4). 
We consider the initial-value problem (2) for the Burgers equation with Riemann initial data $u_{0}$ and final time $T$. That is,

$$
\left\{\begin{array}{l}
u_{t}+u u_{x}=0, \quad(x, t) \in \mathbb{R} \times(0, T), \\
u(x, 0)=u_{0}(x):= \begin{cases}1, & x<T \\
3, & x \geq T .\end{cases}
\end{array}\right.
$$

It is easy to verify that the entropy solution is

$$
u(x, t)= \begin{cases}1, & x<t+T, \\ (x-T) / t, & t+T \leq x<3 t+T, \\ 3, & x \geq 3 t+T,\end{cases}
$$

and another weak solution is

$$
w(x, t)=u_{0}(x-2 t)= \begin{cases}1, & x<2 t+T \\ 3, & x \geq 2 t+T\end{cases}
$$

We define a sequence of mesh sizes $h_{k} \rightarrow 0$ as

$$
h_{k}:=\frac{2 T}{2 k+1}, \quad k=0,1, \ldots,
$$

and set $t_{n k}:=n \Delta t_{k}, n=0, \ldots, N_{k}$, where $\Delta t_{k}:=h_{k} / 2$ and $N_{k}=T / \Delta t_{k}=2 k+1$. The motivation for the particular choice of $h_{k}$ is that the point $x=T$ (which is the point of discontinuity of $\left.u_{0}\right)$ is the midpoint of the interval $I_{k}=\left[k h_{k},(k+1) h_{k}\right)$. Since, from now on, we work with a fixed mesh size, we suppress the index $k$ in the notation.

We now define a projection $P_{h}$ as follows. If $g$ is nondecreasing on $I_{j}$, we define

$$
\left.P_{h} g(x)\right|_{I_{j}}:=\left\{\begin{array}{cc}
\operatorname{essinf} g, & x<y_{j} \\
I_{j} & \\
\operatorname{esssup} g, & x \geq y_{j} \\
I_{j}
\end{array}\right.
$$

whereas, if $g$ is nonincreasing on $I_{j}$,

$$
\left.P_{h} g(x)\right|_{I_{j}}:=\left\{\begin{array}{cc}
\underset{I_{j}}{\operatorname{esssup} g,} & x<y_{j} \\
I_{j} & x \geq y_{j} \\
\underset{I_{j}}{\operatorname{esinf}} g, & x
\end{array}\right.
$$

where the $y_{j}$ are chosen so that $\frac{1}{h} \int_{I_{j}} P_{h} g(x) d x=g_{j}=\frac{1}{h} \int_{I_{j}} g(x) d x, j \in \mathbb{Z}$. Moreover, if $g$ is not monotone on $I_{j}$, then we set $\left.P_{h} g(x)\right|_{I_{j}}:=g_{j}$.

The projection $P_{h}$ and the scheme that it generates satisfy all conditions of Theorem 2 except (P4). Indeed, $P_{h}$ preserves the class $\mathcal{W}_{L}$ for any $L \in \mathbb{N}$ (in fact, it is also nonoscillatory), is conservative, total variation bounded, and local, but, as can be easily seen, $P_{h}$ is not relaxed entropic. For example, if $g(x)=x \chi_{[0, h)}(x), x \in$ $\mathbb{R}$, then $|g|_{\operatorname{Lip}\left(1, L^{1}\right)}=2 h$ and the left-hand side of (11) is $h^{2} / 4$ for $\lambda=h / 2$ and $j=0$. Hence (11) holds for $\alpha=0$ only and does not hold for positive $\alpha$.

Next, we show that the Godunov-type method associated with the projection $P_{h}$ does not converge to the entropy solution $u$. In fact, we prove that the method converges to the weak solution $w$. 
Let $E_{\Delta t}$ be the exact evolution operator, i.e., $v\left(\cdot, t_{n}^{-}\right)=E_{\Delta t} v\left(\cdot, t_{n-1}\right)$. Since $P_{h} u_{0}=u_{0}$ and $v\left(\cdot, t_{n}\right)=P_{h} v\left(\cdot, t_{n}^{-}\right)$, we have $v\left(\cdot, t_{n}\right)=\left(P_{h} E_{\Delta t}\right)^{n} u_{0}$. Moreover, it can be verified (cf. (34)) that

$$
E_{\Delta t} u_{0}= \begin{cases}1, & x<\Delta t+T, \\ (x-T) / \Delta t, & \Delta t+T \leq x<3 \Delta t+T, \\ 3, & x \geq 3 \Delta t+T .\end{cases}
$$

After one time step, we obtain $v\left(\cdot, t_{1}\right)=P_{h} E_{\Delta t} u_{0}=u_{0}(\cdot-h)$. Note that the discontinuity of $u_{0}(\cdot-h)$ is at the midpoint of $I_{k+1}$. Therefore, by induction, we arrive at $v\left(\cdot, t_{n}\right)=u_{0}(\cdot-n h)=w\left(\cdot, t_{n}\right), n=1, \ldots, N$. This means that the numerical solution $v$ at all times $t_{n}$ is precisely the weak solution $w$, i.e., $v(\cdot, T)$ does not converge to the entropy solution as $N \rightarrow \infty$.

The above example is instructive in that it shows that there is no convergence to the entropy solution even if $P_{h}$ is "almost entropic". More precisely, for each time $t_{n}$, inequality (11) is satisfied for all $I_{j}, j \neq k+n$, with $g=v\left(\cdot, t_{n}^{-}\right)$and $C_{1}=0$, i.e., $P_{h}$ is entropic (with this choice of $g$ ) on $I_{j}, j \neq k+n$. In addition, inequality (11) holds for $I_{k+n}$ with $\alpha=0$. Hence, the projection $P_{h}$ violates (P4) just once for

each time $t_{n}$, yet the corresponding numerical solution still does not converge to the "correct" solution.

Finally, we note that, for the sake of simplicity, a noncompactly supported function $u_{0}$ is used in the previous example. However, the above arguments can be readily applied to the initial condition $u_{0} \chi_{[0, m T]}$ (with $m$ large enough), which has a compact support.

\section{REFERENCES}

1. F. Bouchut, Ch. Bourdarias and B. Perthame, A MUSCL method satisfying all entropy inequalities, Math. Comp. 65 (1996), 1439-1461. MR 97a:65080

2. F. Bouchut and B. Perthame, Kružkov's estimates for scalar conservation laws revisited, Trans. Amer. Math. Soc. 350 (1998), no. 7, 2847-2870. MR 98m:65156

3. Y. Brenier and S. Osher, The one-sided Lipschitz condition for convex scalar conservation laws, SIAM J. Numer. Anal. 25 (1988), 8-23. MR 89a:65134

4. P. Colella and P. Woodward, The piecewise parabolic method for gas-dynamical simulations, J. Comput. Phys. 54 (1984), 174-201.

5. F. Coquel and P. LeFloch, An entropy satisfying MUSCL scheme for systems of conservation laws, Numer. Math. 74 (1996), 1-33. MR 97g:65179

6. R. A. DeVore and G. G. Lorentz, Constructive Approximation, Springer-Verlag, Berlin, 1993. MR 95f:41001

7. A. Harten and S. Osher, Uniformly high order accurate nonoscillatory schemes, I, SIAM J. Numer. Anal. 24 (1987), no. 2, 279-309. MR 90a:65198

8. A. Harten, B. Enquist, S. Osher and S.R. Chakravarthy, Uniformly high order accurate essentially nonoscillatory schemes, III, J. Comput. Phys. 71 (1987), no. 2, 231-303. MR 90a:65199

9. G.-S. Jiang, C.-T. Lin, S. Osher and E. Tadmor High-resolution nonoscillatory central schemes with nonstaggered grids for hyperbolic conservation laws, SIAM J. Numer. Anal. 35 (1998), no. 6, 2147-2169. MR 99j:65145

10. G.-S. Jiang and E. Tadmor Nonoscillatory central schemes for hyperbolic conservation laws, SIAM J. Sci. Comput. 19 (1998), no. 6, 1892-1917. MR 99f:65128

11. S.N. Kruzhkov, First order quasilinear equations in several independent variables, Math. USSR Sbornik 10 (1970), no. 2, 217-243. MR 42:2159

12. N.N. Kuznetsov, Accuracy of some approximate methods for computing the weak solutions of a first order quasi-linear equations, USSR Comput. Math. and Math. Phys. 16 (1976), no. 6, 105-119. MR 58:3510

13. P. Lax and B. Wendroff, Systems of conservation laws, Comm. Pure Appl. Math. 13 (1960), 217-237. MR 22:11523 
14. X. Liu and S. Osher, Nonoscillatory high order accurate self-similar maximum principle satisfying shock capturing schemes, I, SIAM J. Numer. Anal. 33 (1996), 760-779. MR 97h:65110

15. X. Liu and E. Tadmor, Third order nonoscillatory central scheme for hyperbolic conservation laws, Numer. Math. 79 (1997), 397-425. MR 99h:65149

16. B. Lucier, Error bounds for the methods of Glimm, Godunov and LeVeque, SIAM J. Numer. Anal. 22 (1985), no. 6, 1074-1081. MR 88a:65104

17. H. Nessyahu and E. Tadmor, The convergence rate of nonlinear scalar conservation laws, SIAM J. Numer. Anal. 29 (1992), 1505-1519. MR 93j:65139

18. S. Osher and E. Tadmor, On the convergence rate of difference approximations to scalar conservation laws, Math. Comp. 50 (1988), 19-51. MR 89m:65086

19. R. Rockafellar and R. Wets, Variational analysis, Springer-Verlag, Berlin, 1998. MR 98m:49001

20. C.-W. Shu, Numerical experiments on the accuracy of ENO and modified ENO schemes, J. Sci. Comput. 5 (1990), no. 2, 127-149.

21. E. Tadmor, Local error estimates for discontinuous solutions of nonlinear hyperbolic equations, SIAM J. Numer. Anal. 28 (1991), 891-906. MR 92d:35190

22. - Approximate solutions of Nonlinear Conservation Laws and Related Equations, Proc. Sympos. Appl. Math., 54 (1998), 325-368. MR 99c:35149

23. W. Ziemer, Weakly Differentiable Functions, Graduate Texts in Mathematics, SpringerVerlag, 1989. MR 91e:46046

Department of Mathematics, University of Manitoba, Winnipeg, Manitoba, R3T 2N2 CANADA

E-mail address: kopotunk@cc.umanitoba.ca

Department of Mathematics, Vanderbilt University, Nashville, Tennessee 37240

E-mail address: neamtu@math.vanderbilt.edu

Department of Mathematics, Texas A\&M University, College Station, Texas 77845

E-mail address: popov@math.tamu.edu 